\title{
DISEÑO DE UN SISTEMA PILOTO DE COSTEO ABC COMO HERRAMIENTA PARA LA TOMA DE DECISIONES EN UNA EMPRESA DE SERVICIOS, EL CASO DE LA AGENCIA ECOTURISMO PUTUMAYO
}

\section{DESIGN OF A PILOT SYSTEM OF ABC COST AS A TOOL FOR DECISION-MAKING IN A SERVICE COMPANY, THE CASE OF THE PUTUMAYO ECOTOURISM AGENCY}

\section{Luis Ángel Meneses Cerón' \\ Linz Shirley Rueda ${ }^{2}$}

\section{RESUMEN}

Actualmente, la gestión de costos en la empresa es un tema relevante en el contexto de los mercados globales. Algunas organizaciones adoptaron el Sistema de Costos Basado en Actividades, ya que aborda de manera eficiente los costos en que incurren para la prestación de servicios, desde una perspectiva que engloba los aspectos económicos relacionados con cada una de las actividades ejecutadas, generando información valiosa que constituye una herramienta de apoyo para la toma de decisiones, permitiendo a las compañías estar a la vanguardia ante los cambios del entorno. El objetivo del trabajo, es diseñar un sistema de costos $A B C$ en la agencia Ecoturismo Putumayo. Al respecto, se utilizó una metodología descriptiva para recolectar la información, procesarla y proponer el sistema de costeo. Finalmente, se logró identificar y estandarizar a través de un modelo piloto, los recursos incurridos en la prestación del servicio, determinando el costo de los paquetes turísticos que la agencia ofrece al mercado.

Palabras clave: sistema de costo, recursos, procesos, actividades, inductores de costo, objeto de costos.

1 Doctorando en Economía de los Negocios, Universidad ICESI. Docente tiempo completo Universidad Cooperativa de Colombia. Correo electrónico: luis.menesesc@campusucc.edu.co Orcid: https://orcid.org/0000-0003-0467-8970

2 Administradora de Empresas de la Corporación Universitaria Autónoma del Cauca y contadora pública de la Universidad del Cauca.

Correo electrónico: lishiru4@hotmail.com

Orcid: https://orcid.org/0000-0002-5899-3055

Código JEL: N26, M41, L83, D24.

Fecha de recepción: 10/04/2018

Fecha de aprobación: 4/09/2018

DOI: https://doi.org/10.18601/16577175.n22.07 


\section{ABSTRACT}

Currently, cost management in the company is a relevant issue in the context of global markets. Some organizations adopted the Activity Based Costing System, since it efficiently addresses the costs incurred for the provision of services, from a perspective that encompasses the economic aspects related to each of the activities carried out, generating valuable information that constitutes a support tool for decisionmaking, allowing companies to be at the forefront in the face of environmental changes. The objective of the work is to design a system of $\mathrm{ABC}$ costs in the Ecoturismo Putumayo agency. In this regard, a descriptive methodology was used to collect the information, process it and propose the costing system. Finally, it was possible to identify and standardize, through a pilot model, the resources incurred in the provision of the service, determining the cost of the tour packages that the agency offers to the market.

Keywords: system cost, resources, processes, activities, and cost driver, cost object.

\section{INTRODUCCIÓN}

El entorno actual en el que se desenvuelven las empresas, se caracteriza por tener que dar respuesta a un mercado altamente competitivo y de constante cambio, dinámica originada principalmente por aspectos como la globalización, el auge de la tecnología, la alta disponibilidad de información, entre otros. En ese contexto, y en la búsqueda de un mejor desempeño económico de la empresa, cada vez adquiere mayor importancia la contabilidad de costos por la relevancia que puede tener la información que de ella se deriva. No obstante, la contabilidad de costos tradicional no se ha desarrollado a la par de los avances tecnológicos, por lo que se ha visto en la urgencia de encontrar nuevas formas de responder a las necesidades de las organizaciones.

Las diferentes organizaciones, independientemente del sector en que se desenvuelven, es decir, primario, secundario y terciario, requieren el desarrollo de competencias específicas en costos que solventen la toma de decisiones a nivel estratégico, táctico y operativo para encausar el éxito empresarial. Lo anterior, exige una función proactiva de la contabilidad de costos, como un área esencial en la búsqueda y desarrollo de ventajas competitivas que garanticen la supervivencia y sostenibilidad de las empresas a través del tiempo.

Constantemente, se señala que el sistema de costos $\mathrm{ABC}$ tiene más exactitud que los sistemas de costos tradicionales, pues es capaz de reconocer en detalle los causantes del costo y ayuda a administrar mejor las actividades operacionales de una compañía. La implementación de un sistema de costeo por actividades requiere que la organización cumpla con una serie de requisitos particulares y los convierta en parte de su cultura de trabajo. Este sistema de costeo sirve como herramienta vital a la gerencia y le permite a esta conocer realmente el costo tanto de sus actividades como de sus productos y servicios para obtener 
de ellos el máximo aprovechamiento posible, reducir los consumos no productivos y sobre todo poder tomar decisiones más efectivas y acordes con la realidad del negocio y del mercado.

Por otra parte, es claro que "el sector turismo no escapa a la realidad de la globalización, siendo necesario manejar información relevante y oportuna en materia de costos, como pieza clave para desarrollar ventajas, cautivando mercados a partir de la relación justa entre valor y precios" (Morillo y Cardozo, 2017, p. 92). En ese orden de ideas, el presente artículo presenta los resultados finales de un trabajo académico, consistente en el diseño e implementación de un sistema piloto de costeo $\mathrm{ABC}$ como herramienta para la toma de decisiones operativas y estratégicas en la agencia Ecoturismo Putumayo. La aplicación del sistema de costos $\mathrm{ABC}$ le permitirá a esta organización del sector de servicios obtener una mejor información sobre sus procesos, optimizar el uso del personal y otros recursos, brindándole una orientación razonable para poder competir en el mercado del servicio turístico, proyectando con éxito a la empresa dentro de la región y a nivel nacional.

Este artículo se encuentra estructurado de la siguiente manera: en la segunda sección se plantea la metodología de la investigación. La tercera sección realiza una revisión de la literatura y los principales aspectos conceptuales. En la cuarta sección se analizan los datos y se muestra los resultados de la investigación y, finalmente, la última sección presenta las conclusiones.

\section{MATERIALES Y MÉTODOS}

El trabajo realizado en la agencia Ecoturismo Putumayo es un estudio de caso aplicado al sector hotelero, que surgió de un análisis crítico del desarrollo de su objeto social, a partir del cual se determinó que una de sus estrategias para alcanzar con oportunidad y eficiencia las metas establecidas por sus directivas era poseer un sistema de costos por actividades, que garantizara una oportuna operatividad en la prestación del servicio y, a su vez, una gestión financiera eficiente de sus recursos de naturaleza privada.

A partir de dicho contexto, surgieron las siguientes preguntas de investigación:

¿Está la organización empleando adecuadamente sus recursos en el área operativa y estos, a su vez, se reflejan en los costos?

¿Permitirá la forma actual de asignar costos, establecer sistemas de control de las operaciones y generar medición de las eficiencias?

¿La empresa está en la capacidad de dar respuesta rápida a cotizaciones? ¿La organización puede responder rápidamente a cambios de precio de la competencia?

¿Cómo puede influir el diseño de un sistema de costos en la planificación de la prestación de los servicios?

¿Con la actual forma de costeo de la organización, se podrán controlar los costos y explicar las razones de sus cambios? 
En ese sentido, se determinó que la investigación es de tipo descriptivo, la cual consistió en estudiar situaciones que se dan en condiciones naturales, por tanto, se estuvo en contacto directo con las actividades operativas de la agencia para recolectar información de tipo administrativa, contable y financiera, atender solicitudes y resolver inquietudes presentadas durante la elaboración del trabajo.

El objetivo de la investigación consistió en especificar las situaciones, costumbres y actitudes predominantes en un contexto particular a través de la descripción exacta de las actividades, objetos, procesos, personas o cualquier otro fenómeno que se someta a un análisis riguroso. Su meta no se limita exclusivamente a la recolección de datos, sino a la predicción e identificación de las relaciones que existen entre dos o más variables. Los investigadores no son meros tabuladores, sino que recogen los datos sobre la base de una hipótesis o teoría, exponen y resumen la información de manera cuidadosa y luego analizan minuciosamente los resultados a fin de extraer generalizaciones significativas que contribuyan al conocimiento (Hernández, Fernández y Baptista, 2014).

\section{Fases de investigación}

La metodología empleada se estructuró a partir de estudios realizados sobre el costeo por actividades (Jung y Dall' Agnol, 2016; Cuervo y Otros, 2013; Madeneira, 2011; Cesarotti y Spada, 2009; Contreras y Mac Cawley, 2006; Cuevas et al., 2004) y ha sido probada con éxito en un considerable número de empresas de diversas características obteniendo resultados muy satisfactorios. $\mathrm{Al}$ respecto, se compone de una serie de pasos que conforman el protocolo para la implementación del modelo $\mathrm{ABC}$, los cuales se describen a continuación.

\section{Caracterización de la empresa}

La caracterización comprende la identificación de todos los factores que intervienen en un proceso y que se deben controlar, por tanto, el desarrollo del sistema de costos requiere un conocimiento integral de la empresa, lo cual se logra mediante el estudio de diversos aspectos tales como: estatutos, plataforma estratégica, objetivos y políticas corporativas, manuales de funciones y procedimientos, portafolio de productos y servicios, áreas de responsabilidad, estructura organizacional, procesos operativos y otros aspectos relevantes que contribuyan a generar una visión clara y objetiva de la compañía y su negocio.

\section{Identificación de actividades}

Una vez conocidos y evaluados los procesos de la empresa, se logran determinar todas las actividades desarrolladas en ellos de tal forma que se pueda pasar de un plano general a uno particular definiendo las (macro) actividades y subactividades de mayor relevancia enmarcadas en el proceso de prestación de servicios turísticos para finalmente evaluar la influencia y el valor agregado de cada una de ellas. Las actividades deben ser diligentemente seleccionadas según la cadena de valor y el mapa de procesos de la empresa y 
absolutamente todas deben ser posteriormente costeadas.

\section{Determinación de generadores de carga}

Los generadores de carga hacen referencia a los empleados que consumen un recurso y, por tanto, generan el costo de una determinada actividad. De acuerdo con el proceso, a cada actividad le corresponde su propio inductor de carga, el cual dependerá del tipo de actividad y del lugar o momento en que se realice. Dado el caso, puede ser más de uno dependiendo de la intensidad y del número de subactividades en la cual se desagregue la actividad principal (Contreras y Mac Cawley, 2006).

\section{Determinación de inductores de costo (cost drivers)}

Dependiendo del número y tipo de las subactividades, los inductores de costo tienden a ser distintos para cada una de ellas, realizando la elección de cada inductor objetivamente según como se consume el recurso y a la forma en que es transferido su costo al generador de carga (actividad) y de este al producto o servicio final (Contreras y Mac Cawley, 2006).

\section{Identificación de recursos}

Los recursos hacen referencia a una relación de elementos (costos y gastos agrupados homogéneamente) que se utilizan en la ejecución de las diferentes actividades operacionales de la empresa. Dado que cada proceso es diferente y por presentar cada actividad sus pro- pios generadores de carga e inductores de costo, la asignación (consumo) de recursos es particular. El total de recursos se identifica y acumula de acuerdo con el proceso, al tipo de servicio y al propósito del estudio, el cual es, simplemente, la imputación de los costos que cada paquete turístico genera durante la prestación del servicio.

\section{Identificación de objetos de costos}

Un objeto de costos es todo aquello para lo cual se desea una medición separada de costos, por ejemplo, un producto, un servicio, una división, un área o, simplemente, un proyecto o un cliente. Cada proceso da origen a objetos diferentes los cuales serán cuantificados, por consiguiente, se identifican los servicios finales que la empresa comercializa y presta, los cuales representan los distintos paquetes turísticos ofertados en el área de influencia.

\section{Asignación de los costos a las actividades}

Cuando se haya determinado objetiva y razonablemente el inductor de costo, se imputa el costo del consumo de algún recurso (costos más gastos) a las subactividades, para determinar el costo total de la actividad ejecutada a un generador de carga. Dicho costo se trasfiere y vincula al proceso, por consiguiente, al servicio final.

\section{Asignación de los costos de las actividades a los servicios}

"De acuerdo al paso anterior, cada actividad tendrá su propio costo y agre- 
gación de valor al proceso" (Contreras y Mac Cawley, 2006). Una vez determinado el costo de cada actividad, se determina el consumo de actividades por parte de los servicios finales y la suma de todas las actividades que intervienen en el proceso de una determinada categoría, formaran el costo total del servicio.

\section{Población y muestra}

El estudio fue realizado en las instalaciones de la empresa durante el segundo semestre del 2016 y para su desarrollo se necesitó la colaboración del personal administrativo constituido por el geren- te, la recepcionista y el mayordomo, así como también de todos los colaboradores operativos de la agencia, quienes suministraron la información necesaria relacionada con las áreas de la empresa e igualmente con las características de los procesos y de los productos y servicios que allí se generan.

\section{Proceso de implementación del modelo piloto de costeo $A B C$}

Una vez determinadas las bases conceptuales del sistema $\mathrm{ABC}$, lo siguiente es su aplicación en la agencia de turismo mediante los siguientes pasos (ver gráfico 1).

\section{Gráfico 1.}

Implementación de un modelo piloto de costeo $\mathrm{ABC}$

Proceso de implementación de un modelo piloto de costeo ABC

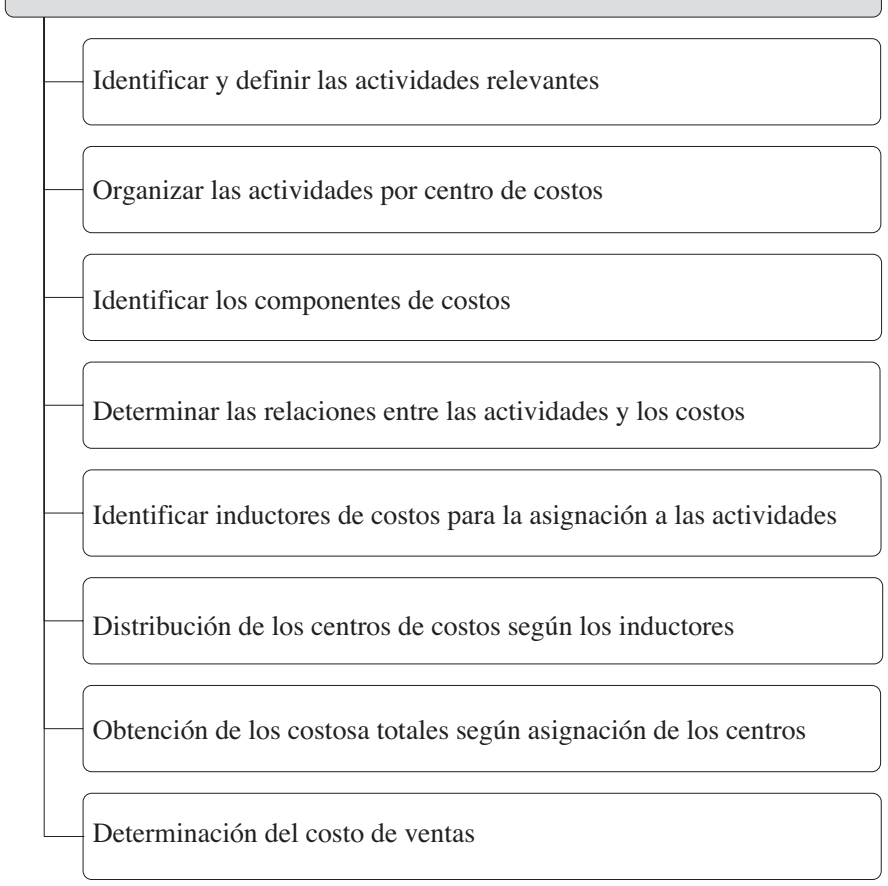

Fuente: elaboración propia. 


\section{ASPECTOS CONCEPTUALES DEL SISTEMA DE COSTEO POR ACTIVIDADES (ACTIVITY BASED COSTING)}

\author{
Pequeña y mediana empresa \\ (pyme)
}

La Junta de Normas Internacionales de Contabilidad (International Accounting Standards Board) (IASB) (2009) precisa como pequeñas y medianas empresas a los entes económicos que no tienen obligación pública de rendir cuentas, es decir, aquellas entidades que según regulación interna, publican sus estados financieros con propósito de información general con la finalidad de brindar información a usuarios externos, entendiéndose estos como los propietarios que no están vinculados en la gestión del negocio, los acreedores actuales o potenciales o las agencias de calificación crediticia.

\section{Empresa de servicios}

Las empresas de servicios son aquellas unidades económicas organizadas para la prestación y venta de actividades, beneficios o satisfacciones humanas, y que son esencialmente intangibles y no dan como resultado la propiedad de algo (Choy, 2012). De acuerdo con la tipología establecida por la economía clásica, las empresas de servicios conforman el sector terciario de la economía.

La determinación de los costos en las empresas de servicios constituye una labor compleja y diversa, ya que los procedimientos de cálculo dependen de varios factores, entre ellos, el tipo de actividad, el modelo de negocio y las características o especificidades de la empresa. Por ende, el proceso de determinar el costo en una empresa de turismo difiere al de una empresa de banca comercial o de servicios de asesoría profesional por citar algunos casos.

\section{Sistema de costos}

Un sistema de costos es un sistema de información derivado de la contabilidad general, encargado de gestionar los detalles referentes al costo total de fabricación de un bien o prestación de un servicio. Al respecto, se compone de un conjunto organizado de criterios y procedimientos para la clasificación, acumulación y asignación de costes a los productos y centros de actividad y responsabilidad con el propósito de ofrecer información relevante para la toma de decisiones y el control (Mori1lo, 2002).

En principio, los sistemas de costeo existentes fueron pensados y diseñados para su aplicación en las empresas industriales o manufactureras; no obstante, hoy vemos cómo diferentes organizaciones, especialmente las de servicios, reconocen la importancia de contar con información veraz y oportuna referente a sus costos de operación. Un sistema de costeo, se compone de tres elementos fundamentales: el método de acumulación de costos, el método de asignación de costos y la base de costeo (Cuervo, Osorio y Duque, 2013).

La acumulación de costos corresponde a un periodo $u$ horizonte temporal determinado, definido en forma particular 
por la empresa, el cual puede ser diario, mensual o anual. La acumulación de costos incluye la recolección organizada de datos de costos mediante un conjunto de procedimientos y técnicas contables y administrativas. Por último, los costos acumulados deberán asignarse a los objetos de costo y del resultado de esta operación se obtendrá el costo unitario promedio de los bienes o servicios. $\mathrm{Al}$ respecto, la contabilidad de costos reconoce la existencia de los siguientes métodos de acumulación de costos: por órdenes de fabricación, procesos, actividades, proyectos y ciclo de vida del producto, entre otros (Garrison, Noreen y Brewer, 2007).

\section{El Costeo Basado en Actividades $(A B C)$}

La utilización de un sistema de gestión y de costos por actividades adquiere especial relevancia en un entorno tan dinámico y turbulento como el imperante a finales del siglo XX (Chacón y Sarmiento, 2003), en el que gerentes y directivos corporativos necesitan información relevante que les permita tomar decisiones efectivas en relación con el diseño de productos y servicios, la inversión en activos, la programación de la producción y los procesos tecnológicos, la logística y comercialización, elementos en su totalidad relacionados directamente con la rentabilidad de la compañía.

En ese orden de ideas, si bien es cierto, en la década del setenta surgieron las primeras aproximaciones teóricas al sistema del Costeo Basado en Actividades (ABC), solo se conocen sus primeras aplicaciones prácticas hasta la década del ochenta, en la medida en que el desarrollo de la tecnología informática y de telecomunicaciones puso a disposición de las empresas los medios suficientes (hardware, software y protocolos) para obtener y procesar más fácilmente información clave para su implementación. Es así como, en 1987, Robert S. Kaplan y Robín Cooper, profesores de la Universidad de Harvard, formalizaron el modelo $\mathrm{ABC}$ por medio de su obra cumbre, Accounting and management: a field study perspective, en la que establecen este nuevo enfoque para calcular y gestionar los costos como herramienta indispensable para poder competir en el ambiente empresarial actual.

El Costeo Basado en Actividades (ABC, por sus iniciales en inglés, Activity $\mathrm{Ba}$ sed Costing), es un sistema de gestión y acumulación de costos denominado de doble fase, puesto que se basa en el hecho de que una empresa para producir bienes y servicios necesita llevar a cabo actividades, las cuales consumen recursos por lo que en primera instancia se costean las actividades y, posteriormente, el costo de las actividades es asignado a los diferentes objetos de costo (producto, servicios, áreas, divisiones, grupos de clientes o proveedores, regiones y procesos, entre otros) que demandan dichas actividades (ver gráfico 2). En ese sentido, la metodología $A B C$ logra una mayor precisión o razonabilidad en la determinación de los costos y de la respectiva rentabilidad de la empresa al superar problemas contables como: las inconsistencias causadas por las distribuciones de costos indirectos, los subsidios de costos (subcosteo 
y sobrecosteo) y costos unitarios poco confiables (Cuevas, 2010).

Gráfico 2.

Esquema de distribución de costos por actividades

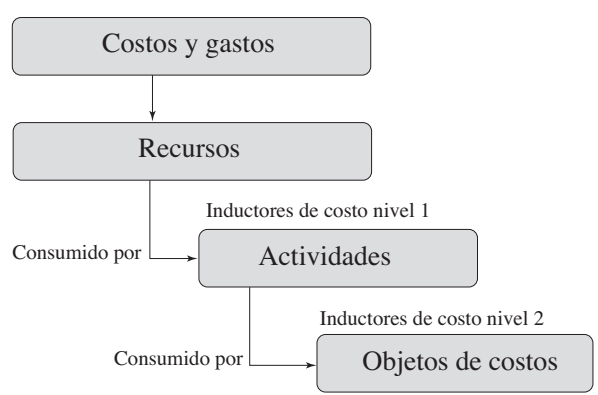

Fuente: elaboración propia.

Con respecto a la metodología $\mathrm{ABC}$, Cuervo et al. identificaron las siguientes características básicas:

- Más que un modelo contable es un modelo gerencial.

- Una empresa para producir productos o prestar servicios necesita llevar a cabo actividades.

- Las actividades consumen recursos.

- Los recursos incluyen tanto los costos como los gastos.

- Los productos o servicios (objetos de costo) consumen actividades.

- Se basa en las relaciones de causalidad entre los recursos y las actividades y entre estas y los objetos de costo.

- Su enfoque es sistémico, es decir son más importantes las actividades y procesos que las divisiones o departamentos. Es la base fundamental para desarrollar la administración basada en actividades.

- Su aplicación exitosa requiere del compromiso de todos los empleados y directivos de la firma.
El modelo de costeo por actividades facilita la medición integral de los costos de la empresa y la comprensión de su comportamiento, a la vez que facilita la toma de decisiones empresariales en múltiples aspectos operativos y estratégicos. García, Rodríguez y Velásquez (2005) identificaron que los aspectos más significativos que permite conocer la metodología $\mathrm{ABC}$ en relación con la empresa son:

- El costo de las diferentes actividades de la empresa.

- El costo de los procesos de negocio.

- El costo de los diferentes componentes de la cadena de valor.

- El costo de los productos o servicios.

- El costo de los clientes de forma individual o colectiva.

- Que productos son rentables y cuáles no.

- Decisiones de hacer o comprar en toda la cadena de valor.

- Reducciones de costos basados en el análisis de las actividades.

- Reducciones de costos basados en el análisis de los procesos.

- La clasificación de las actividades en actividades con valor agregado y sin valor agregado.

- Decisiones sobre reducción, eliminación o división de determinadas actividades.

Finalmente, desde la perspectiva contextual, es relevante afirmar que en Colombia vienen adoptándose políticas públicas y privadas en torno al mejoramiento de la calidad de la información contable y la inclusión del costeo por actividades en dicha información, siendo el sector público 
pionero en la materia a través de una transformación hacia modelos operativos de libre competencia basados en la autofinanciación y autosostenibilidad. Algunas investigaciones al respecto (Contaduría General de la Nación, 2008; Jara, Castañeda y Gómez, 2002; Osorio, 2000) reconocen que desde hace más de una década sectores como el de servicios públicos domiciliarios $\mathrm{y}$ salud han venido desarrollando y perfeccionando sistemas de costos en las empresas que componen estos sectores. Al respecto, se pueden citar los siguientes antecedentes:

- Según la Resolución 2863 del 9 de octubre de 1996, por la cual se adopta el sistema de costos y gastos para las empresas de telecomunicaciones.

- Según la Resolución 1417 del 18 de abril de 1997, por la cual se expide y adopta el Sistema Unificado de Costos y Gastos para entes prestadores de servicios públicos domiciliarios de acueducto, alcantarillado, aseo, energía y gas combustible.

- La normatividad emitida para este sector fue revisada y actualizada en diciembre del 2005.

- En el caso de las entidades prestadoras de servicios de salud se ha establecido la obligatoriedad de contar con sistemas de costos en las empresas tanto públicas como privadas, según lo estipulado en la Ley 100 de 1993. A nivel de costos, se efectuó una primera aproximación en el 2001, a través de la elaboración e implementación del Manual de procedimientos administrativos para la implementación de un sistema de contabilidad de costos hospitalarios, tomando como base la metodología implementada por el Ministerio de Salud.

- Además, la contaduría general de la nación en el 2008, dentro de la serie de textos de contabilidad pública, emitió el documento marco de referencia para la implantación del sistema de costos en las entidades del sector público.

\section{DISEÑO E IMPLEMENTACIÓN DEL SISTEMA PILOTO DE COSTEO ABC EN LA AGENCIA ECOTURISMO PUTUMAYO}

Con el fin de delinear el sistema de costos a aplicar en la empresa objeto de estudio y la consecuente metodología para calcular los costos de sus servicios es necesario conocer en profundidad su estructura administrativa y operacional.

\section{Aspectos generales de la agencia de turismo Ecoturismo Putumayo}

La agencia de turismo Ecoturismo Putumayo fue constituida bajo la idea emprendedora del señor Juan Pablo Ramírez, actual propietario y gerente, quien analizando el impactante incremento de turistas nacionales e internacionales en la región decidió crear una microempresa dedicada a la exploración de sitios turísticos en el departamento del Putumayo, para ello contó con la ayuda de campesinos de la región, que con gran esfuerzo lograron sacar adelante uno de los negocios con mayor proyección en el departamento. Acto seguido, con el registro nacional de turismo n..$^{\circ} 27599$ se le dio vida jurídica como agencia de viajes y a través 
del registro n. $^{\circ} 27748$ empezó a operar como establecimiento de alojamiento, hospedaje, vivienda y turística conformando La Posada Turística Dantayaco; sin lugar a dudas, estas dos iniciativas sentaron los cimientos para el desarrollo en el sector turístico de esta pyme (ver gráfico 3).

Gráfico 3.

Estructura organizacional de Ecoturismo Putumayo

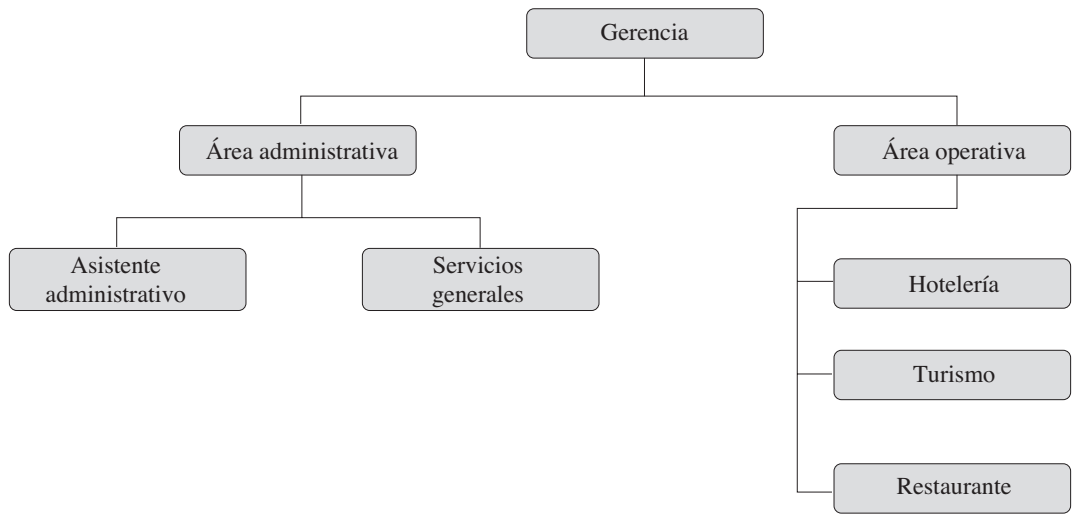

Fuente: elaboración propia, 2017.

Actualmente, Ecoturismo Putumayo cuenta con un portafolio de servicios que se adapta a las necesidades de los turistas, los cuales pueden disfrutar de una forma distinta e innovadora de servicio, una presencia constante y estratégica de publicidad por internet e importantes alianzas con empresas del sector, lo cual ha permitido que hoy la agencia sea conocida a nivel nacional e internacional.

\section{Misión}

Ecoturismo Putumayo, agencia y operadora turística del departamento del Putumayo, es una empresa que cuenta con el personal idóneo y capaz de recibir turistas nacionales e internacionales en sus instalaciones para hacerlos disfrutar de los diferentes servicios que ofrece. Actualmente, es una de las empresas con mayor proyección en la región.
Visión

Ecoturismo Putumayo, para el 2020, pretende ser una agencia de viajes y operadora establecida y con un gran reconocimiento a nivel nacional e internacional, aumentando su portafolio de servicios e infraestructura para que con innovación logre que el departamento del Putumayo sea uno de los destinos preferidos de los turistas que visitan el país.

\section{Portafolio de servicios}

\section{Posada turística Dantayaco}

La posada turística Dantayaco es un hospedaje diseñado en un ambiente familiar en medio de la naturaleza. Se encuentra ubicado a cien metros antes de la entrada al atractivo turístico Fin del Mundo, km 6 vía Mocoa - Villa Garzón, Putumayo. 
Desde este lugar, se puede acceder a otros atractivos turísticos de la ciudad de Mocoa, entre ellos: El Fin del Mundo, Mariposario Paway, Ojo de Dios, Parque Temático Suruma, Hornoyaco, Cavernas de Licamancha, Centro de Estudio Amazónicos (CEA), Cañón del Mandiyaco, Serranía de los Churumbelos y Canalendres.

\section{Actividades relevantes de la agencia Ecoturismo Putumayo}

Después de realizar la aplicación de un cuestionario preestablecido a través de una entrevista con la totalidad del personal operativo y administrativo de la agencia, se obtuvieron las siguientes actividades (ver cuadro 1).

Cuadro 1.

Actividades

\begin{tabular}{|c|l|}
\hline \multicolumn{2}{|c|}{ Ecoturismo Putumayo - Costeo ABC } \\
\hline \multicolumn{2}{|c|}{ Lista de chequeo de actividades } \\
\hline Cód. & \multicolumn{1}{|c|}{ Actividades } \\
\hline 1 & Atención a clientes. \\
\hline 2 & Contestar y realizar llamadas telefónicas. \\
\hline 3 & Registrar reservas. \\
\hline 4 & Registrar huéspedes. \\
\hline 5 & Elaboración de cotizaciones y envío. \\
\hline 6 & Revisión de email, páginas y redes sociales. \\
\hline 7 & Arqueo de caja. \\
\hline 8 & Limpieza habitaciones. \\
\hline 9 & Lavado de ropa, sabanas y cobijas. \\
\hline 10 & Secado de ropa, sabanas y cobijas. \\
\hline 11 & Planchado. \\
\hline 12 & Cuidado y mantenimiento general. \\
\hline 13 & Compras suministros. \\
\hline 14 & Orientación de sitios turísticos. \\
\hline 15 & Traslado a sitios turísticos. \\
\hline
\end{tabular}

Fuente: elaboración propia basado en Ecoturismo Putumayo, 2017.
Actividades y relación con los centros de costos

Según lo analizado en la estructura organizacional de la agencia se identificaron tres centros de costos operativos: hotel, restaurante y turismo, así como dos centros de costos de apoyo: recepción, mantenimiento y cuidado, cada uno relacionado con las siguientes actividades (ver cuadro 2).

\section{Componentes de los costos} principales

Para realizar el costeo por metodología $\mathrm{ABC}$, en la agencia se identificaron cinco componentes del costo (recursos), que serán asignados de la siguiente forma: el componente de sueldos y salarios como mano de obra directa, los componentes de cargos fijos, de servicios básicos y de suministros como gastos generales (costos indirectos de fabricación [CIF]) y el último componente como materia prima directa (ver cuadro 3 ).

\section{Relaciones entre componentes del costo y centros de costos principales}

Para una mejor asignación de los recursos consumidos por la agencia a los centros de costos que esta posee, se determinó que los diferentes componentes de costo son generados en varios centros de costos, por tanto, las relaciones de causalidad son las siguientes (ver cuadro 4). 
Cuadro 2.

Relación entre actividades y centros de costos

\begin{tabular}{|c|l|l|l|}
\hline \multicolumn{3}{|c|}{ Lcoturismo Putumayo - costeo ABC } \\
\hline \multicolumn{2}{|c|}{ Actividades } & \multicolumn{2}{c|}{ Centros de costo } \\
\hline \multirow{2}{*}{ Cód. } & \multicolumn{1}{|c|}{ Operativo } & \multicolumn{1}{c|}{ De apoyo } \\
\hline & & & Recepción \\
\hline 1 & Atención a clientes & & Recepción \\
\hline 2 & Contestar y realizar llamadas telefónicas & & Recepción \\
\hline 3 & Registrar reservas & & Recepción \\
\hline 4 & Registrar huéspedes & & Recepción \\
\hline 5 & Elaboración de cotizaciones y envío & & Recepción \\
\hline 6 & Revisión de correo electrónico, páginas y redes sociales & & Recepción \\
\hline 7 & Arqueo de caja & Hotel & \\
\hline 8 & Limpieza habitaciones & Hotel & \\
\hline 9 & Lavado de ropa, sabanas y cobijas & Hotel & \\
\hline 10 & Secado de ropa, sabanas y cobijas & Hotel & \\
\hline 11 & Planchado & & Mantenimiento y cuidado \\
\hline 12 & Cuidado y mantenimiento general & & Mantenimiento y cuidado \\
\hline 13 & Compras suministros & Turismo & \\
\hline 14 & Orientación de sitios turísticos & Turismo & \\
\hline 15 & Traslado a sitios turísticos & & \\
\hline
\end{tabular}

Fuente: elaboración propia basado en Ecoturismo Putumayo, 2017.

Cuadro 3.

Componentes del costo

\begin{tabular}{|l|l|}
\hline \multicolumn{2}{|c|}{ Ecoturismo Putumayo - costeo ABC } \\
\hline \multicolumn{2}{|c|}{ Componentes del costo } \\
\hline 1 & Componentes del costo de sueldos y salarios \\
\hline & Sueldos y salarios \\
\hline & Aportes sociales \\
\hline 2 & Aportes parafiscales \\
\hline & Componentes del costo de cargos fijos \\
\hline 3 & Depreciaciones \\
\hline & Componentes del costo de servicios básicos \\
\hline & Agua potable \\
\hline & Energía eléctrica \\
\hline & Combustibles \\
\hline 4 & Telefonía móvil \\
\hline & Componentes del costo de suministros \\
\hline & Generales de limpieza \\
\hline 5 & Generales operativos \\
\hline & Componentes del costo de materia prima \\
\hline Fuente: elaboración propia basado en Ecoturismo & Menú diario \\
\hline Putumayo, 2017. \\
\hline
\end{tabular}

\section{Cuadro 4.}

Relación entre costos y centros de costos

\begin{tabular}{|l|l|}
\hline \multicolumn{2}{|c|}{ Ecoturismo Putumayo - costeo ABC } \\
\hline \multicolumn{2}{|c|}{ Relación entre costos y centros } \\
\hline Salarios y sueldos & Recepción \\
\hline & Hotel \\
\hline & Mantenimiento y cuidado \\
\hline Depreciaciones & Turismo \\
\hline & Recepción \\
\hline & Restaurante \\
\hline & Hotel \\
\hline & Mantenimiento y cuidado \\
\hline Agua potable & Turismo \\
\hline & Recepción \\
\hline & Restaurante \\
\hline & Hotel \\
\hline & Mantenimiento y cuidado \\
\hline Energía Eléctrica & Turismo \\
\hline & Recepción \\
\hline & Restaurante \\
\hline
\end{tabular}




\begin{tabular}{|l|l|}
\hline & Hotel \\
\hline & Mantenimiento y cuidado \\
\hline & Turismo \\
\hline Combustibles & Recepción \\
\hline & Restaurante \\
\hline & Hotel \\
\hline & Mantenimiento y cuidado \\
\hline & Turismo \\
\hline Telefonía móvil & Recepción \\
\hline Suministros de limpieza & Recepción \\
\hline & Restaurante \\
\hline & Hotel \\
\hline & Mantenimiento y cuidado \\
\hline & Turismo \\
\hline Suministros operativos & Hotel \\
\hline
\end{tabular}

\section{Inductores de costos o cost drivers}

Debido a la forma de operar la agencia, se obtuvo que para la asignación de los costos a los centros, cada gasto general (CIF) tendrá un inductor primario, el cual será imputado de acuerdo con el consumo del recurso efectuado por cada centro, dando como resultado lo siguiente (ver cuadro 5).

Fuente: elaboración propia basado en Ecoturismo Putumayo, 2017.

\section{Cuadro 5.}

Inductores de costos o cost drivers

\begin{tabular}{|c|c|c|}
\hline \multicolumn{3}{|c|}{ Ecoturismo Putumayo } \\
\hline \multicolumn{3}{|c|}{ Lista de chequeo - inductores } \\
\hline Concepto & Inductor & Unid. de medida \\
\hline \multicolumn{3}{|l|}{ Depreciaciones } \\
\hline Edificio & Metros cuadrados & 314 \\
\hline Camarotes & n. ${ }^{\circ}$ camarotes & 22 \\
\hline Nocheros & n. ${ }^{\circ}$ nocheros & 11 \\
\hline Closet & n. ${ }^{\circ}$ closet & 11 \\
\hline Escritorio para oficina & n. ${ }^{\circ}$ escritorio para oficina & 1 \\
\hline Silla para oficina & n. ${ }^{\circ}$ silla para oficina & 1 \\
\hline Portátil & $\mathrm{n} .^{\circ}$ de equipos PC & 1 \\
\hline Impresora tinta continua & n. ${ }^{\circ}$ de equipos & 1 \\
\hline Hamacas & n. ${ }^{\circ}$ hamacas & 4 \\
\hline Juego de sala & n. ${ }^{\circ}$ juego de sala & 1 \\
\hline Televisor & n. ${ }^{\circ}$ televisor & 1 \\
\hline Equipo de sonido & n. ${ }^{\circ}$ equipo de sonido & 1 \\
\hline Estante para tv y sonido & n..$^{\circ}$ estante & 1 \\
\hline Mesa comedor & n. ${ }^{\circ}$ comedor & 2 \\
\hline Bancas comedor & n. ${ }^{\circ}$ bancas & 4 \\
\hline Suministros de limpieza & Metros cuadrados & 314 \\
\hline Suministros operativos & n. ${ }^{\circ}$ clientes & 68 \\
\hline \multicolumn{3}{|l|}{ Servicios públicos } \\
\hline Agua potable & Metros cuadrados & 314 \\
\hline Energía eléctrica & Kilo watts & 266 \\
\hline Combustibles & Metros cuadrados & 314 \\
\hline Telefonía móvil & n. ${ }^{\circ}$ clientes & 68 \\
\hline
\end{tabular}

Fuente: elaboración propia basado en Ecoturismo Putumayo, 2017. 
Asignación de los costos según inductores a los centros de costos

Para distribuir los recursos a los centros de costos y de ahí a los objetos de costo fue necesario establecer el siguiente flujo contable (ver gráficos 4 y 5 ) para determinar el orden de imputación.

Gráfico 4.

Flujo de costos

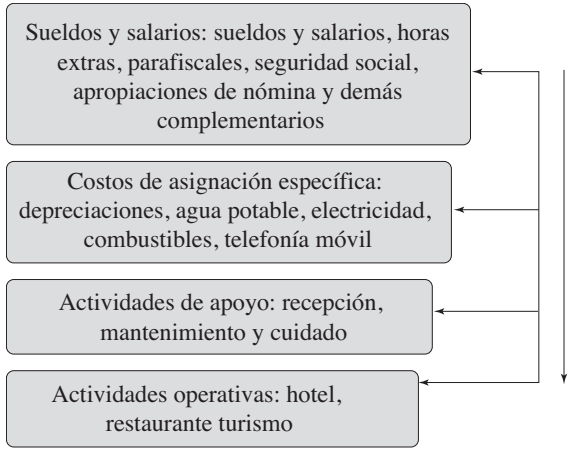

Fuente: elaboración propia basado en información de Ecoturismo Putumayo.

\section{Obtención de costos totales según asignación de los centros de costo}

La asignación por medio de los inductores dio como resultado la acumulación de los costos en cada centro de costo y luego mediante porcentaje de participación o consumo se asignaron a los centros de apoyo y a los centros operativos dando como resultado lo siguiente (ver cuadros 6 y 7):

\section{Determinación de los costos de ventas por servicio y paquetes turísticos}

Para finalizar el proceso de asignación de costos bajo la metodología de $\mathrm{ABC}$, y según el proceso de acumulación que se desarrolló en la agencia de turismo Ecoturismo Putumayo, se encontró el costo de cada servicio y de cada paquete turístico (ruta turística + alimentación +

Gráfico 5.

Metodología adaptada a Ecoturismo Putumayo

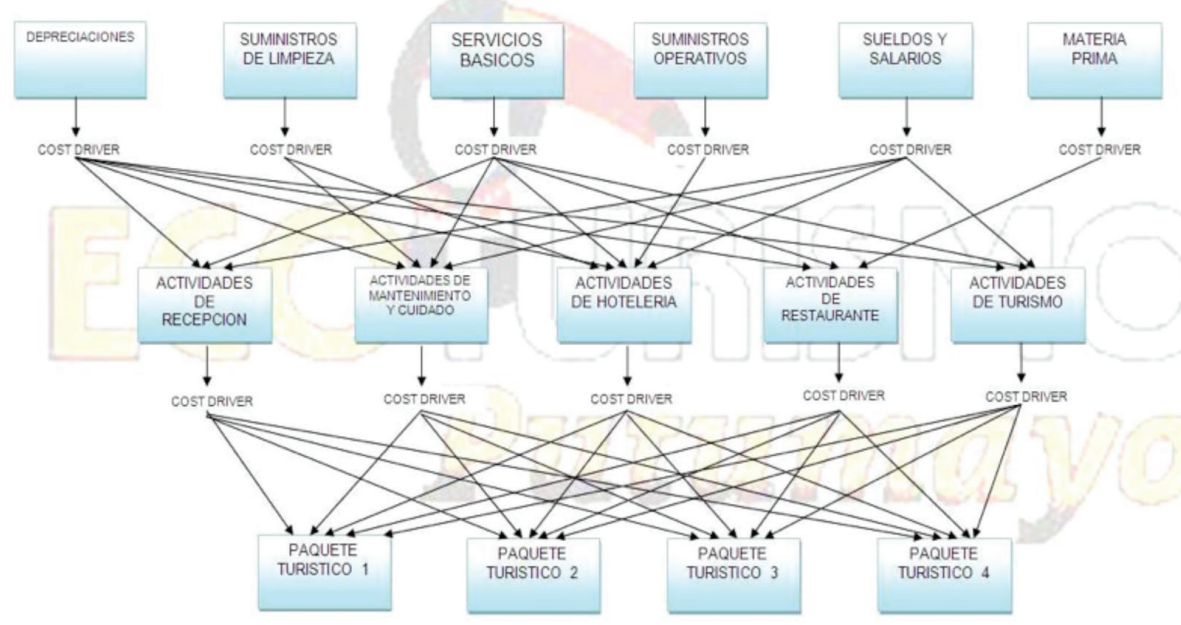


Cuadro 6.

Costos totales en centros de apoyo y operativo

\begin{tabular}{|l|r|r|r|r|}
\hline \multicolumn{5}{|c|}{ Ecoturismo Putumayo } \\
\hline \multicolumn{5}{|c|}{ Elementos del costo } \\
\hline & Mod & \multicolumn{1}{|c|}{ Mp } & \multicolumn{1}{c|}{ Cif } & Mod + mp + cif \\
\hline Centro operativo & $\$ 1011477$ & & $\$ 159045$ & $\$ 1170522$ \\
\hline Hotel & & $\$ 1088000$ & $\$ 28850$ & $\$ 1116850$ \\
\hline Restaurante & $\$ 340000$ & & $\$ 29724$ & $\$ 369724$ \\
\hline Turismo & Subtotal & & $\$ 2657096$ \\
\hline \multicolumn{5}{|c|}{} \\
\hline Centro de apoyo & $\$ 1011477$ & $\$ 84065$ & $\$ 1095541$ \\
\hline Recepción & $\$ 1011477$ & $\$ 4777$ & $\$ 1016254$ \\
\hline Mantenimiento y cuidado & Subtotal & & $\$ 2111795$ \\
\hline
\end{tabular}

Fuente: elaboración propia basado en Ecoturismo Putumayo, 2017.

\section{Cuadro 7.}

Costos totales

\begin{tabular}{|l|r|}
\hline \multicolumn{2}{|c|}{ Ecoturismo Putumayo } \\
\hline \multicolumn{2}{|c|}{ Costos totales } \\
\hline Centros operativos & Total costo $(\$)$ \\
\hline Hotel & 1874453 \\
\hline Restaurante & 1820782 \\
\hline Turismo & 1073655 \\
\hline Total & 4768891 \\
\hline
\end{tabular}

Fuente: elaboración propia basado en Ecoturismo Putumayo, 2017.

hospedaje + transporte + entrada) (ver cuadros 8 y 9 ).

\section{Análisis e interpretación de los} resultados obtenidos

La identificación y asignación de costos para la agencia de Turismo Ecoturismo Putumayo dio como resultado que, asignando todos los costos mediante la metodología del costeo ABC, cada servicio prestado por la agencia tiene los siguientes costos: hotel con un costo de $\$ 27565$, restaurante con un costo de $\$ 26776$ y turismo con un costo de $\$ 15789$, consolidando el valor de un paquete turístico de $\$ 70130$ a cualquier ruta elegida por el cliente sin incluir el transporte (desde el hotel hasta el inicio de la ruta) y la entrada, valores que al ser incluidos en el costo de cada paquete turístico se obtendría lo siguiente:

- Ruta Cañón Mandiyaco y Cavernas de Licamancha por un valor de $\$ 73130$.

- Ruta Hormoyaco y Canalendres por un valor de \$71 130 .

- Ruta Fin del Mundo y Ojo de Dios por un valor de $\$ 72130$.

- Centro experimental amazónico y Mariposario Paway por un valor de \$74131.

Para obtener el total de cada centro de costo (hotel, restaurante y turismo) se tuvo en cuenta algunos de los siguientes aspectos: 
Cuadro 8.

Costo por servicio prestado

\begin{tabular}{|l|c|c|c|}
\hline \multicolumn{4}{|c|}{ Ecoturismo Putumayo } \\
\hline \multicolumn{4}{|c|}{ Total costo por servicio según ventas } \\
\hline Centros operativos & Total costo $(\$)$ & Ventas & Costo (\$) \\
\hline Hotel & 1874453,78 & 68 & 27565 \\
\hline Restaurante & 1820782,16 & 68 & 26776 \\
\hline Turismo & 1073655,68 & 68 & 15789 \\
\hline Total & & 70130 \\
\hline
\end{tabular}

Fuente: elaboración propia basado en Ecoturismo Putumayo, 2017.

Cuadro 9.

Costo por ruta turística

\begin{tabular}{|l|c|c|c|c|}
\hline \multicolumn{5}{|c|}{ Ecoturismo Putumayo } \\
\hline \multicolumn{2}{|c|}{ Costo de ventas y utilidad unitaria } \\
\hline Paquete turístico x día & $\begin{array}{c}\text { Costo } \\
(\$)\end{array}$ & $\begin{array}{c}\text { Transporte } \\
(\$)\end{array}$ & $\begin{array}{c}\text { Entrada } \\
(\$)\end{array}$ & $\begin{array}{c}\text { C. De venta } \\
(\$)\end{array}$ \\
\hline Cañón Mandiyaco y Cavernas Licamancha & 70130 & 3000 & & 73130 \\
\hline Hornoyaco y Canalendres & 70130 & 1000 & & 71130 \\
\hline Fin del Mundo y Ojo de Dios & 70130 & & 2000 & 72130 \\
\hline Cea y Mariposario & 70130 & & 4000 & 74130 \\
\hline
\end{tabular}

Fuente: elaboración propia basado en Ecoturismo Putumayo, 2017.

- Ecoturismo Putumayo es una empresa creada en el régimen simplificado, por tanto, no está obligada a tener contabilidad, lo que quiere decir que la obtención de los datos se hizo mediante registros internos guardados en la documentación, entrevistas y soportes.

- El valor del edificio fue hallado mediante el concepto de costo histórico (valor en libros o escritura pública más todas las afectaciones (modificaciones y construcciones) que aumente el valor del activo.

- El valor de la gran mayoría de los muebles y enseres de la agencia fueron valorados a precio de mercado de artículos artesanales, ya que estos fueron elaborados por los campesinos de la región siguiendo las especificaciones de producción dadas por el gerente quien logró darle proyección a la organización bajo el concepto de ecoturismo.

- La depreciación de los activos fijos se hizo mediante el método de línea recta según lo acordado con el gerente de la agencia.

- Los servicios básicos (agua y energía eléctrica) tienen un costo mensual constante, ya que el hotel está ubicado en una vereda del municipio de Mocoa y, por tanto, no existe algún tipo de contador que regule el consumo. 
- Se aclara que la agencia Ecoturismo Putumayo no elabora los productos ofrecidos en el restaurante, si no que contrata a campesinos de la región para que hagan este proceso de producción, la agencia solo compra y vende obteniendo un margen de utilidad por eso al centro de costo del restaurante solo se asignan los gastos generales correspondientes y el valor del menú diario como materia prima directa.

- Para el valor de las ventas de los paquetes turísticos se tomó la información brindada por el gerente, quien es el que fija los precios, haciendo un promedio de las ventas registradas durante cuatro meses, tomando temporadas altas y bajas, ya que los precios variaban de una a otra temporada y según la demanda presentada.
- Para efectos de presupuestos y con previa autorización del gerente se hizo una proyección anual del estado de rentas y gastos, para lo cual se tomó la decisión de que para el paquete turístico CEA y Mariposario se modificará el precio de venta (actualmente se encuentra por debajo del costo) teniendo en cuenta que su margen de rentabilidad será del $10 \%$ (promedio del margen de los tres paquetes turísticos) quedando con un precio de venta aproximado de $\$ 84000$ y se mantendrá el mismo nivel de ventas durante todo el año.

A partir de la información anterior, se estructuró el estado de resultados proyectado para la agencia, precisando un mayor grado de detalle, al expresarlo por objeto de costo y por actividades (ver cuadro 10 y 11).

Cuadro 10.

Estado de resultados proyectado

\begin{tabular}{|l|c|c|c|}
\hline \multicolumn{4}{|c|}{ Ecoturismo Putumayo - costo ABC } \\
\hline \multicolumn{4}{|c|}{ Estado de rentas y gastos anual (presupuestado) } \\
\hline Ventas & Cant. & Precio $(\$)$ & Total $(\$)$ \\
\hline Cañón Mandiyaco y Cavernas Licamancha & 132 & 90000 & 11880000 \\
\hline Hornoyaco y Canalendres & 180 & 82000 & 14760000 \\
\hline Fin del Mundo y Ojo de Dios & 276 & 78000 & 21528000 \\
\hline Cea y Mariposario & 228 & 84000 & 19152000 \\
\hline Total ingresos & Cant. & Costo (\$) & Total (\$) \\
\hline Costo de Ventas & 132 & 73131 & 9653260 \\
\hline Cañón Mandiyaco y Cavernas Licamancha & 180 & 71131 & 12803537 \\
\hline Hornoyaco y Canalendres & 276 & 72131 & 19908090 \\
\hline Fin del Mundo y Ojo de Dios & 228 & 74131 & 16901813 \\
\hline Cea y Mariposario & & 59266700 \\
\hline Total costo & & 8053300 \\
\hline Utilidad
\end{tabular}

Fuente: elaboración propia a partir de información de Ecoturismo Putumayo, 2017. 
Cuadro 11.

Estado de rentas y gastos mensual por actividades (presupuestado)

\begin{tabular}{|l|c|c|c|}
\hline \multicolumn{4}{|c|}{ Ecoturismo Putumayo - costo ABC } \\
\hline \multicolumn{3}{|c|}{ Estado de rentas y gastos mensual (presupuestado) por actividades } \\
\hline \multicolumn{1}{|c|}{ Ventas } & Cant. & Precio (\$) & Total (\$) \\
\hline Cañón Mandiyaco y Cavernas Licamancha & 11 & 90000 & 990000 \\
\hline Hornoyaco y Canalendres & 15 & 82000 & 1230000 \\
\hline Fin del Mundo y Ojo de Dios & 23 & 78000 & 1794000 \\
\hline Cea y Mariposario & 19 & 84000 & 1596000 \\
\hline Total ingresos & & & 5610000 \\
\hline Costo de ventas por actividades & Cant. & Costo $(\$)$ & Total $(\$)$ \\
\hline Atención a clientes & 6760 & 105,53 & 713395 \\
\hline Contestar y realizar llamadas telefónicas & 780 & 105,53 & 82314 \\
\hline Registrar reservas & 1300 & 105,53 & 137191 \\
\hline Registrar huéspedes & 1300 & 105,53 & 137191 \\
\hline Elaboración de cotizaciones y envío & 780 & 105,53 & 82314 \\
\hline Revisión de email, páginas y redes sociales & 780 & 105,53 & 82314 \\
\hline Arqueo de caja & 780 & 105,53 & 82314 \\
\hline Limpieza habitaciones & 6240 & 105,53 & 658518 \\
\hline Lavado de ropa, sabanas y cobijas & 1560 & 105,53 & 164629 \\
\hline Secado de ropa, sabanas y cobijas & 1560 & 105,53 & 164629 \\
\hline Planchado & 3120 & 105,53 & 329259 \\
\hline Cuidado y mantenimiento general & 9360 & 105,53 & 987778 \\
\hline Compras suministros & 3120 & 105,53 & 329259 \\
\hline Orientación de sitios turísticos & 1560 & 105,53 & 164629 \\
\hline Traslado a sitios turísticos & 7800 & 105,53 & 823148 \\
\hline Total costo & & & 4938891 \\
\hline Utilidad & & 671108 \\
\hline
\end{tabular}

Fuente: elaboración propia a partir de información de Ecoturismo Putumayo, 2017.

\section{CONCLUSIONES}

El diseño e implementación del modelo piloto de costeo $\mathrm{ABC}$ fue vital importancia para la agencia Ecoturismo Putumayo, puesto que permitió establecer un costo certero de los servicios prestados a sus clientes. Adicionalmente, ayudó a solucionar la problemática tradicional de la distribución de los costos indirectos de fabricación (CIF), disyuntiva en la cual se había visto inmersa la empresa a través del tiempo. En ese sentido, el resultado obtenido en la aplicación de la metodología de costeo por actividades $\mathrm{ABC}$ muestra cifras más acertadas, que influyen directamente no solo en la fijación del precio de venta del servicio, sino en la estrategia de comercialización.

El sistema de costos $A B C$ fue diseñado con la finalidad de que los administradores cuenten con una herramienta para la gestión estratégica de sus costos, permitiendo la reducción de estos y la efectividad en la toma de decisiones al brindar información más exacta y deta- 
llada de los procesos y actividades permitiendo avances en la estructuración de la ventaja competitiva y mejorando el enfoque de servicio al cliente en aras de incrementar la creación de valor para los accionistas. En concordancia con Morillo y Cardozo (2017), la presente investigación identificó que en el caso del sector hotelero, el ABC permite precisar con mayor certeza y detalle la composición de los costos asociados a cada uno de los servicios ofertados. Lo anterior, permite impulsar aquellos servicios de mayor rentabilidad, en medio de condiciones complejas como la alta competitividad, la estacionalidad de las ventas, la abundancia y variedad de costos fijos, variables e indirectos, entre otras.

Es claro que las medianas y pequeñas empresas que innovan sus sistemas o estructuras de costos pueden mejorar su gestión a largo plazo. En ese sentido, el ABC permite analizar holísticamente los procesos de negocios, detectar actividades y productos que no generan valor e incrementar la rentabilidad al tomar mejores decisiones vinculando practicas gerenciales modernas como el benchmarking, outsourcing, gestión de inventarios, mezcla de ventas, diseño de nuevos productos, entre otras. No obstante, tal como lo afirma Masoudi, Cudney y Paryani (2013), su implementación presenta ciertas barreras o limitaciones, entre ellas, la falta de apoyo de los directivos, el alto costo de la implementación, limitaciones de tiempo, el desconocimiento del sistema de costeo y las diferencias en el proceso de implementación según el sector, industria o negocio.
Los sistemas de costos y control de gestión paulatinamente han venido cobrando importancia en el dinámico mundo empresarial actual y paralelamente, en el entorno de la investigación contable, en aspectos como el diseño, implementación y evaluación en las organizaciones. En Colombia, se requiere apoyar el crecimiento de la pyme y considerar la importancia que tiene la contabilidad de costos y la contabilidad de gestión como herramienta para que este tipo de empresas sean eficientes, efectivas y logren un crecimiento sostenible a largo plazo. En el futuro, este trabajo puede resultar de interés para que los profesionales en contabilidad, finanzas y administración reflexionen sobre la oportunidad que tienen de apoyar a las organizaciones que demandan profesionales capacitados en sistemas innovadores de gestión y control como el sistema de costos $\mathrm{ABC}$.

\section{REFERENCIAS BIBLIOGRÁFICAS}

Bermeo, J. y Bermeo, E. (2005). Las directrices del costo como fuentes de ventajas competitivas. Estudios Gerenciales, (94), pp. 81-106. Recuperado de https://www.icesi.edu. co/revistas/index.php/estudios_gerenciales/article/view/153

Cesarotti, V. y Spada, C. (2009) A systemic approach to achieve operational excellence in hotel services. International Journal of Quality and Service Sciences, 1, issue 1, pp. 51-66. Recuperado de https://doi. org/10.1108/17566690910945868

Chacón, G y Sarmiento, J. (2003). Propuesta de una metodología para la implantación del costeo basado en 
la actividad en la hotelería cubana. Experiencias prácticas alcanzadas. Ponencia presentada en el II Evento Internacional Contabilidad y Finanzas en el Tercer Milenio, La Habana, Cuba.

Contreras, H. y Mac Cawley, A. (2005). Implementacion de un modelo de costos $\mathrm{ABC}$ en una empresa vitivinícola. Economia Agraria, (10), 25-36. Recuperado de https://ageconsearch. umn.edu/bitstream/97353/2/Contreras\%20et\%20al.pdf

Cuervo, J., Osorio, J. y Duque, M. (2013). Costeo basado en actividades $A B C$, gestión basada en actividades $A B M$. Bogotá: ECOE ediciones.

Cuevas, C. (2010). Contabilidad de costos. Bogotá: Pearson Educación.

Cuevas, C., Chávez, G., Castillo, J., Caicedo, N. y Solarte, W. (2004). Costeo ABC. ¿Por qué y cómo implantarlo? Estudios Gerenciales, (92), pp. 47104. Recuperado de https://www. icesi.edu.co/revistas/index.php/estudios_gerenciales/article/view/143

Choy, E. (2012). El dilema de los costos en las empresas de servicios. Revista de la Facultad de Ciencias Contables,(20), 7-14. Recuperado de http://revistasinvestigacion.unmsm. edu.pe/index.php/quipu/article/ view/3852/3085

García, A., Rodríguez, L. y Velásquez, L. (2005). Modelo conceptual del sistema de costos ABC para COOFINEP Cooperativa Financiera (Tesis de especialización inédita). Universidad de Medellín, Medellín, Colombia.

Garrison, R., Noreen, E.y Brewer,P. (2007). Contabilidad administrativa. México: McGraw Hill Interamericana.
Hernández, R., Fernández, C. y Baptista,P. (2014). Metodología de la investigación. México: McGraw Hill.

Jara, G., Castañeda, J. y Gómez, L. (2003). Sistema de costeo basado en actividades como herramienta del presupuesto inteligente para Santafé de Bogotá D. C. Colombia. Revista de Contaduría, (1), fasc.: 43, 179-204. Recuperado de https://aprendeenlinea.udea.edu.co/revistas/index.php/ cont/article/download/25663/2118 $6+\& \mathrm{~cd}=3 \& \mathrm{hl}=\mathrm{es} \& \mathrm{ct}=\mathrm{clnk} \& \mathrm{gl}=\mathrm{co}$

Jung, P. y Dall'Agnol, R. (2016). Price setting in hotel management: a case study. Revista Turismo, (18), issue: 1, 107-133. Recuperado de https: https://siaiap32.univali.br//seer/

Masoudi, A., Cudney, E. y Paryani, K. (2013). Customer-driven hotel landscaping design: A case study. International Journal of Quality \& Reliability Management, (30), 832-852. Recuperado de https:// www.researchgate.net/publication/263120768_Customer-driven_hotel_landscaping_design_A_ case_study

Morillo, M. (2002). Diseño de sistemas de costeo: fundamentos teoricos. Revista Actualidad Contable FACES, 5(5), 7-22. Recuperado de http://www.redalyc.org/articulo.oa? $\mathrm{id}=25700507$

Morillo, M. y Cardozo, C. (2017). Sistema de costos basado en actividades en hoteles cuatro estrellas del estado Mérida, Venezuela. Revista Innovar Journal, 27(64), 91-114. Recuperado de https://revistas.unal. edu.co/index.php/innovar/article/ view/62371/60522

Osorio, J. (2000). Costeo basado en actividades para las empresas de 
acueducto, alcantarillado, aseo, energía, gas. En I Encuentro Iberoamericano de Contabilidad de Gestión (pp. 1-13). Valencia, España: Universidad de Valencia. Recuperado de http://www.observatorio-iberoamericano.org/paises/ spain/art\%C3\%ADculos\%20diversos $\% 20$ sobre $\% 20$ contabilidad $\% 20$ de $\% 20$ gesti\%C3\%B3n/i\%20encuentro\%20iberoamericano $\% 20$ cont. $\% 20$ gesti $\%$ C3\%B3n/modelos\%20abc\%20abm/jairosorio.pdf Textos de contabilidad pública. (2008). Marco de referencia para la implantación del sistema de costos en las entidades del sector público. Bogotá: Contaduría General de la Nación. Recuperado de http://portalterritorial.gov.co/apc-aa-files/75 15a587f637c2c66d45f01f9c4f315c/ marocrefencia_sistcostos.pdf 